\title{
Bilder einfrieren, oder: Wie das Bewegtbild in den Eisschrank kommt
}

Aktuell ist das Einfrieren von Bildern eine sehr alltäglich gewordene Erfahrung, die in Videochats bzw. -konferenzen regelmäßig als Störung auftritt und zu irritierenden Momenten in der Kommunikation führen kann. Im Kachelraster der pandemiesicheren Videokonferenz zeigen sich Teilnehmer*innen immer wieder in unterschiedlichen Zeitverhältnissen: ${ }^{1}$ Manche Gesichter auf diesen Kacheln frieren temporär ein, um dann unvermittelt in einem Time Lapse ${ }^{2}$ die Zeit wieder einzuholen oder auch ganz aus der Konferenz herauszufallen.

In der Regel liegt diese spontan auftretende Dysfunktionalität an einer nicht ausreichenden Bandbreite des Netzes oder an einer Überlastung der Streaming-Server. Das Bild wird nicht mehr übertragen und das letzte vorhandene aus dem Arbeitsspeicher als eingefrorenes Bild wiederholt. Ein derartiger Glitch ist mit seiner NichtIntentionalität anders einzuordnen als die intentionalen Freeze Frames des Films, die ihn aus narrativen, dramaturgischen, ästhetischen oder analytischen Gründen auf einem Bild anhalten lassen. ${ }^{3}$

Im Kontext des Filmbildes wird der Begriff „Freeze Frame“ nachweislich seit 1948 für ein virtuell stehendes Bild verwendet. ${ }^{4}$ Es ist zu vermuten, dass aber schon der Film It's a Wonderful Life (Frank Capra, 1946) mit dem paradigmatischen Einfrieren James Stewards zu Beginn des Films für die Etablierung des Begriffs „Freeze“ gesorgt hat. Im Drehbuch zu diesem Film ist jedenfalls vom Einfrieren des Bildes die Rede: „Suddenly, in action, as George stands with his arms outstretched in illustration, the picture freezes and becomes a still." ${ }^{5}$ Umgangssprachlich leuchtet heute sofort ein, warum bewegte Bilder eingefroren werden können. Das direkte Anhalten (frz.: arrêt sur image) auf dem Bild scheint einer Erstarrung ähnlich, die durch Einfrieren (engl.: freeze) ausgelöst wird: Etwas Bewegtes wird hier unmittelbar stillgestellt. Es soll hier darüber hinaus argumentiert werden, dass erst mit dem Film die Rede vom Einfrieren der Bilder sinnvoll erscheint. Nur in der Differenz zur Bewegung - also zur vergehenden Zeit - kann ein abruptes Anhalten als ein Einfrieren wahrgenommen und auch so bezeichnet werden.

1 „During the months of lockdown, our networked screenactivity took place - and for many still does mainly in the domesticsphere, unfolding ambivalences of proximity and distance, protection and distant (re-)socializing.“ Olga Moskatova: Networked Screens: Topologies of Distance and Media Regime of Immunization. In: img journal, 2020, Nr. 2, S. 282-305.

2 Der Ausdruck leitet sich von lat. lapsus (Ausrutscher) her und deutet an, dass die Zeit in dem beschleunigten Bild ausrutscht, was zugleich eine Metapher für das rasche Vergehen von Zeit ist.

3 Vgl. Torben Sangild: Glitch-The beauty of malfunction. In: Christopher J. Washburne, Maiken Derno (Hg.): Bad music: The Music We Love to Hate, London 2004, S. 257-274.

4 Der Freeze Frame wird nach Merriam Webster zum ersten Mal 1948 im Kontext des Filmes verwendet, zu Beginn des Kalten Krieges, wobei dieser Zusammenhang eher zufällig sein dürfte, vgl. www. merriam-webster.com/dictionary/freeze-frame (Stand 3/2021).

5 Drehbuch von Frances Goodrich, Albert Hackett, Frank Capra und Jo Swerling, 1946, S. 19. 


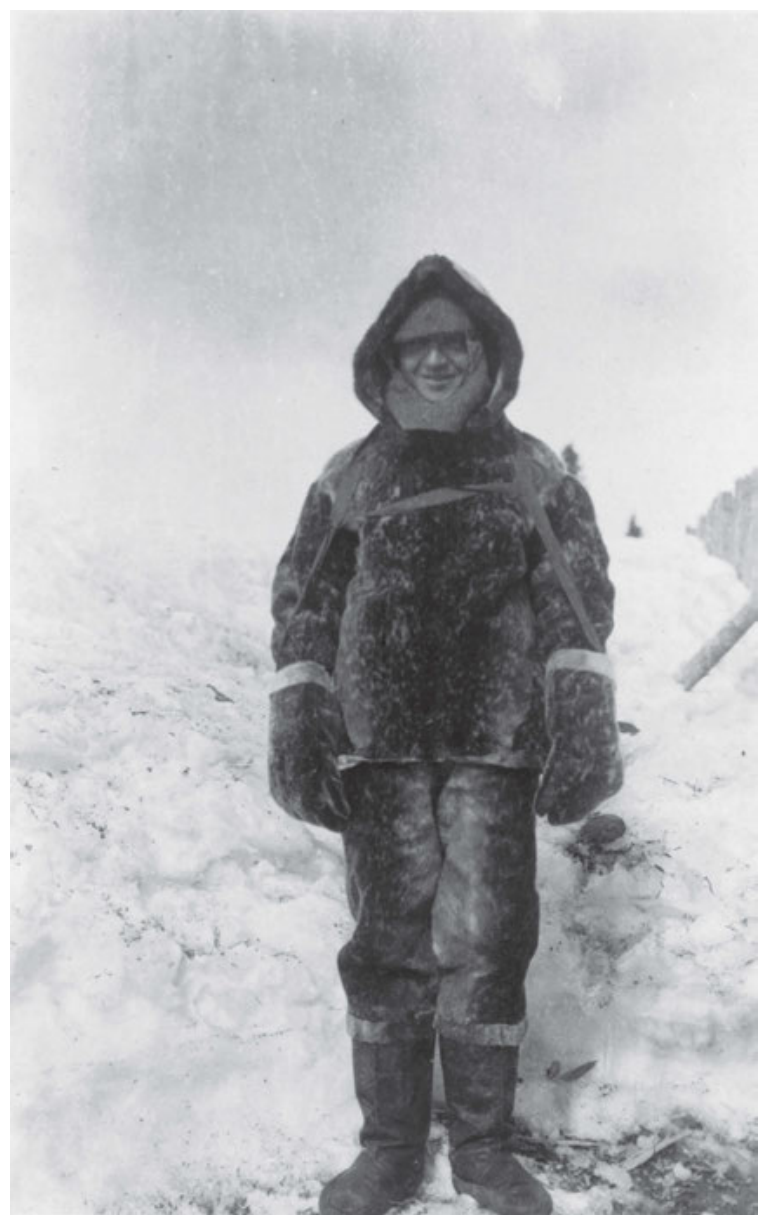

1: Clarence Birdseye in Labrador, Kanada.

\section{Schockfrostung}

Es scheint außerdem, dass eine Kulturtechnik die metaphorische Übertragung des Begriffs vorbereitet. Das Kühlen oder Einfrieren von Lebensmitteln zu deren Erhalt ist seit Jahrtausenden bekannt; in China wurden schon vor ca. 3.000 Jahren die ersten Eiskeller gebaut. Massentauglich mechanisiert wurde das Kühlen von Lebensmitteln im 19. Jahrhundert durch Carl von Linde.

Besonders relevant aber ist Clarence Birdseyes Erfindung des Verfahrens der maschinellen Schockfrostung. Als junger Biologe in Labrador, einer östlichen Provinz des heutigen Kanadas, lernte er von den Inuit das Einfrieren von Fisch. $\pi$ Abb. 1 Sie schockfrosteten ihre Beute, die sie aus Löchern im Eis gezogen hatten, indem sie den Fisch dem Wind und einer Temperatur von ca. $-40^{\circ} \mathrm{C}$ aussetzten. Birdseye bemerkte, dass der Fisch beim späteren Auftauen nicht breiig wurde wie andere Tiefkühl-

kost, die er zuvor probiert hatte. Dies lag an der Geschwindigkeit des Einfrierens, weil langsames Einfrieren die Eiskristalle in den Zellen wachsen lässt, welche deren Struktur zerstören. 1927 meldete Birdseye daraufhin das Patent ${ }^{6}$ für eine Mehrplatten-Gefriermaschine an. $\rtimes$ Abb. 2 Er platzierte Lebensmittel bei $-25^{\circ} \mathrm{C}$ zwischen zwei Metallplatten gegen einen niedrigen Konvektionstunnel, um sie schockzufrieren. ${ }^{7}$

6 US Patent \#1,773,079.

7 Alfonos Totosaus: Frozen Meat - Packaging and Quality Control. In: Y. H. Hui et al. (Hg.): Handbook of Frozen Foods, New York/Basel 2004, S. 227-231, hier S. 227. 1928 gelang Birdseye die Entwicklung des Doppelbandgefrierers, dem Vorläufer der modernen Gefriertechnik. 1930 wurde die erste Tiefkühlproduktlinie durch die Birds Eye Frosted Food Company vermarktet. 


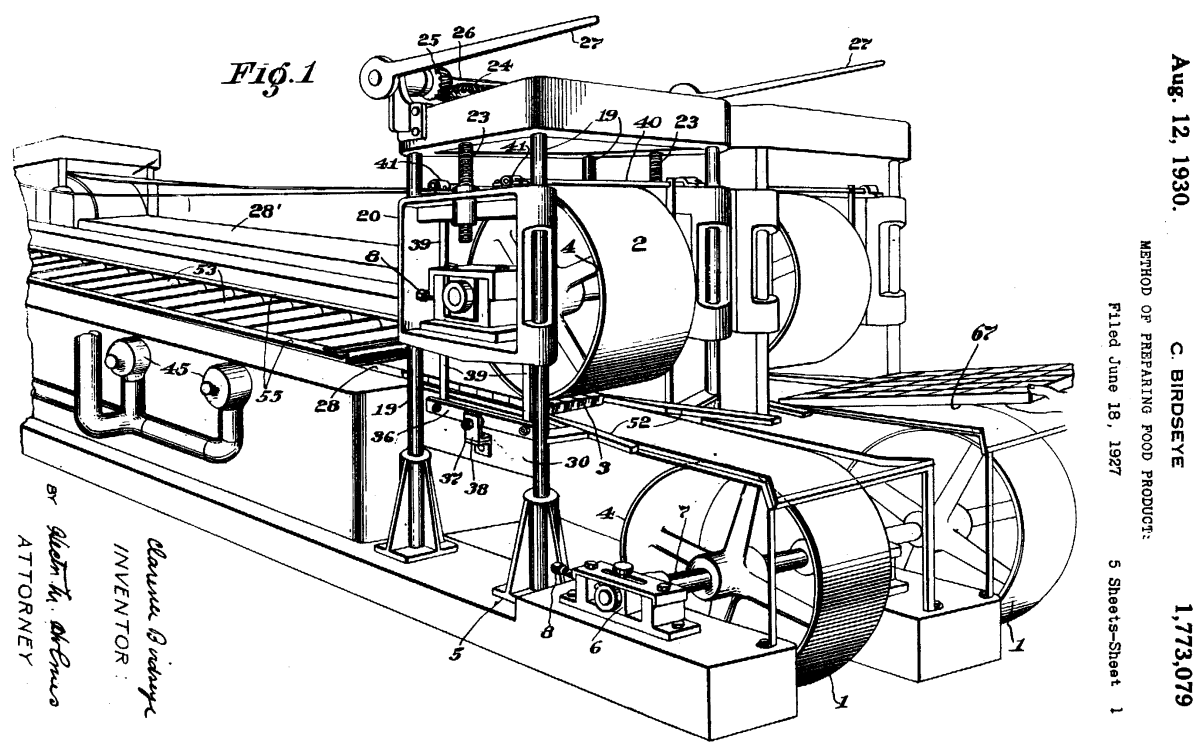

2: Clarence Birdseye: Patent für eine Mehrplatten-Gefriermaschine, 1930 [1927].

Diese Entwicklung findet ungefähr zeitgleich mit den ersten Einsätzen von sogenannten Freeze Frames im Film statt. Es ist noch nicht untersucht, aber denkbar, dass hier ein Zusammenhang besteht. Frühe, populär gewordene Einsätze des Freeze Frame finden sich beim russischen Avantgardisten Dziga Vertov in Der Mann mit der Kamera (Chelovek s kino-apparatom, 1929) त Abb. 3 und in René Clairs Paris qui dort (1924). Vertov hält ein Pferd, das eine Kutsche zieht, im Freeze - eine Standkopie ${ }^{8}$ des Filmbildes - fest und verweist so auf die Basis des Films, die Fotografie. ${ }^{9}$ Clair wendet den Freeze dagegen narrativ: Der Film handelt von einem verrückten Arzt, der einen magischen Strahl auf Menschen anwendet und sie in seltsamen und oft peinlichen Positionen erstarren lässt. ${ }^{10}$

8 Stehkader: „Standkopierung (Freeze Frame). Ein eingefrorenes Filmbild, das durch Mehrfachkopierung eines Einzelbildes auf der optischen Bank erzeugt wird.“ James Monaco: Film verstehen, Reinbek 2017, S. 231. Die kürzest mögliche Einstellung des Freeze ist im analogen Film ein Einzelbild lang, die maximal mögliche Länge einer Einstellung ist durch die Größe des verwendeten Filmmagazins begrenzt. Das sind ca. 11 Minuten bei einem herkömmlichen 35-Millimeter-Filmmagazin.

9 Vielleicht auch ein versteckter Hinweis auf die von Muybridge foto-experimentell geklärte Frage, ob ein Pferd im Galopp gleichzeitig alle vier Läufe in der Luft hat.

10 Das tut er allerdings, indem sich die Schauspieler*innen in den Szenen nicht bewegen. 


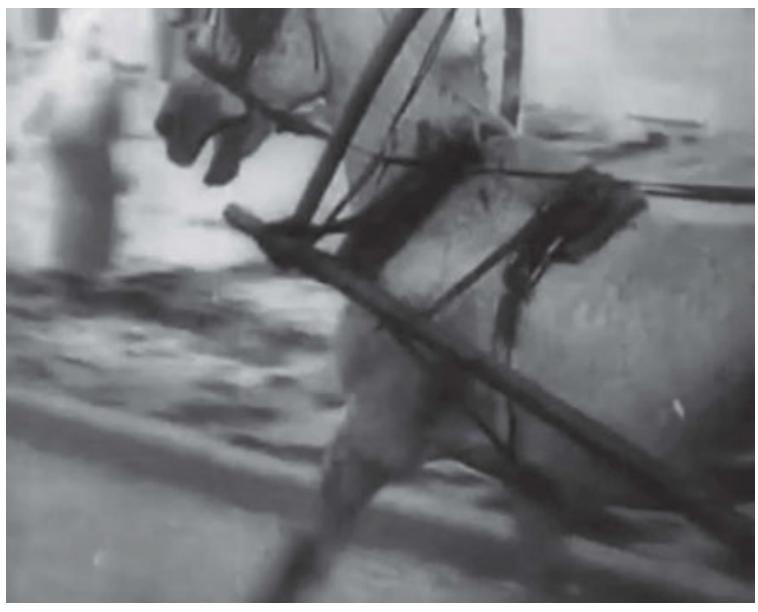

3: Dziga Vertov: Man with the Movie Camera, 1929, Film-Still, 67 min., TC: 23:08.
Für die frühe experimentelle Phase des Films beschreibt Raymond Bellour den Einsatz des Freeze Frame als eine der Möglichkeiten, Filmzeit zu erschließen:

„Yet, it seems that the freeze-frame was at the time only one way among many to manage a film-time that was obsessed with conquering its movements. One can see it as a form, admittedly extreme, yet similar to other processes such as slow and fast motion, reverse motion, and so on. " 11

\section{Ausgestellter Stillstand}

Es könnte vermutet werden, dass die Geschichte des Festhaltens von Bewegung so lang ist wie die Geschichte des Bildes, aber bei genauerem Hinsehen haben die meisten bildlichen Darstellungen bis in das 19. Jahrhundert schon vor der Bildwerdung tendenziell stillgestanden; sie sind nicht erst im oder durch das Bild eingefroren worden, sondern schon zuvor in der Pose erstarrt. Die ästhetische Theorie hat daraus einige wesentliche Beobachtungen zum glücklichen Moment der Darstellung oder der Darstellbarkeit von Handlung abgeleitet. Sogar die frühe Fotografie stellt aufgrund der langen Belichtungszeiten ihre aufzunehmenden Subjekte still, indem sie diese in Halterungen zwängt oder ihnen Möglichkeiten zum Verweilen und Aufstützen bietet. Bewegte Objekte können unterdessen komplett verschwinden, wie in den Langzeitbelichtungen der frühen Daguerro- oder Kalotypien, oder in einem nebulösen Licht aufgehen.

Die wenigen Ausnahmen eines eingefroren wirkenden Stillstandes in der Kunst beginnen erst im Barock: Es sind Gian Lorenzo Bernini und Hendrick Goltzius, die auf unterschiedliche Weise das abrupte Stillstellen von Bewegung thematisieren. Bernini vollzieht dies in der multiperspektivischen Skulptur, deren Protagonist*innen sich im Moment größter Anspannung in den Raum ausdehnen (David, 1623-1624) oder sich im Moment einer spontan festgehaltenen Bewegung verwandeln (Daphne, von 
Apollo verfolgt, 1622-1625). ${ }^{12}$ Goltzius dagegen hält seine vier bekannten Himmelsstürmer (1588) im freien Fall fest und schafft hiermit die ersten Bilder eines ausgestellten Stillstands, der an ein Einfrieren der Figur (im Unterschied zum Einfrieren eines Bildes) denken lässt. ${ }^{13} \rtimes$ Abb. 4 Diese Bilder zeigen den Moment einer nicht-vollendeten Zukunft. ${ }^{14} \mathrm{Im}$ Gegensatz dazu verarbeitet die klassizistische Skulptur den Stillstand im kalten Marmor als ,dauerhaftes körperhaftes Dasein und ihre härtere Materialkonsistenz hat sich vor der Ewigkeit zu bewähren“. ${ }^{15}$ Sie treibt das momenthaft Stillgestellte der Skulptur Berninis mit der Venus Victrix Canovas (1805-1808) aus.

Mit Jean Siméon Chardins Seifenblasen (ca. 1733/34) gerät dieser besondere Moment wieder in den Blick, als eine Blase, die von kürzester Lebensdauer ist und deren Zukunft absehbar, aber unsichtbar ist. Mit der Serie bzw. Sequenz erfährt dann ein weiteres Motiv des Stillstellens besondere Aufmerksamkeit: Claude Monets $\mathrm{Heu}$ haufen (1888-91) und seine mehrfachen Ansichten der Kathedrale von Rouen (1892/93)

12 Sie könnten so als eine Vorwegnahme der sogenannten Bullet Time angesehen werden, die multiperspektivische Figur ist in der Bewegung erstarrt und kann im Raum und in der Zeit erschlossen werden. Viel treffender als Bullet Time sind die Bezeichnungen der frühen Entwickler dieses Effekts - Dayton Taylor und Tim Macmillan -, sie nennen ihn Time Slice oder Frozen-Time-Effekt. Vgl. Winfried Gerling: Die eingefrorene Zeit. In: Stefanie Diekmann, ders. (Hg.): Freeze Frames - Zum Verhältnis von Fotografie und Film, Bielefeld 2009, S. 146-170, hier S. 156-162.

13 Vgl. Winfried Gerling und Fabian Goppelsröder: Was der Fall ist... Prekäre Choreographien, Berlin 2017, S. 12f. Interessant ist, dass diese Bilder anscheinend erst mit der (Wieder-)Entdeckung der Unendlichkeit und des Falls der Erde auf die Sonne bzw. des gravitativen Zusammenwirkens der Sonne mit den Planeten möglich werden.

14 Im Unterschied zu Barthes, der in jeder Fotografie das Bild einer vollendeten Zukunft sieht: „[...] das wird sein und das ist gewesen“ und „Ich lese gleichzeitig: das wird sein und das ist gewesen; mit Schrecken gewahre ich eine vollendete Zukunft, deren Einsatz der Tod ist“, Roland Barthes: Die helle Kammer. Bemerkung zur Photographie. Frankfurt a. M. 2016, S. 86 bzw. S. 106.

15 Vgl. Christa Lichtenstern: „Der Marmor lacht nicht“. Beobachtungen zu Diderots Verständnis der Skulptur. In: Wallraf-Richartz-Jahrbuch, 1987/88, 48/49, S. 269-297, hier S. 282. 
zeugen von der zeitlichen Veränderung der Wahrnehmung, die hier mit einer Konzentration auf Licht und Farbwirkung einhergeht und dadurch über Paul Cézanne in die Abstraktion führt. Marcel Duchamps Akt, eine Treppe herabsteigend (1912) und Pablo Picassos kubistische Violinen erzeugen in der Folge eine sukzessive Multiperspektivität, in der die Zeit verräumlicht ist.

\section{Sekundenbruchteile}

Die meisten fotografischen Verfahren beruhen auf dem Prinzip der Camera obscura, wobei schon im 19. Jahrhundert, als Fotokameras noch nicht besonders mobil waren, darauf hingewiesen wurde, dass sie ein Medium der Bewegung bzw. der Darstellung der bewegten Natur sei. Diderot und d'Alembert notieren unter dem Stichwort „Kammer“, dass die Camera obscura Farben und Bewegung wie kein anderes Medium darstelle. ${ }^{16}$ Die Ausschnitthaftigkeit der Projektion wurde allerdings als eine Konzentration der Wahrnehmung beschrieben, welche die Natur als Ereignis inszeniert. ${ }^{17}$ Mit dieser Vorgeschichte etablierten sich im Diskurs der frühen Fotografie Begriffe wie „Zeichnen“, „Fixieren“"19 und „Spontaneität“" ${ }^{20}$ und nicht etwa „Einfrieren“. So existiert auch kein fotografischer Fachbegriff, der dem Freeze Frame oder dem Frozen-Time-

16 Chambre. In: Denis Diderot, Jean le Rond d'Alembert (Hg.): Encyclopédie ou Dictionnaire raisonné des Sciences, des Arts et des Métiers, Bd. 3, Paris 1751-1765, S. 45-65, hier S. 62.

17 Siehe hierzu Anita Hosseini: Die Experimentalkultur in einer Seifenblase. Das epistemische Potenzial in Chardins Malerei, Paderborn 2017, S. 188-203.

18 „It was during these thoughts that the idea occurred to me ... how charming it would be if it were possible to cause these natural images to imprint themselves durably, and remain fixed upon the paper!“ Henry Fox Talbot: The Pencil of Nature, London 1844, S. 4. Bei Talbot geht es um zwei Formen der Fixierung: Einerseits das Festhalten des Bildes, das in der Camera obscura erscheint, und andererseits das langfristige Fixieren (Erhalten) des fotografischen Bildes: „[...] it followed that a picture made with the chloride could be fixed by dipping it into a bath of the alkaline iodide“, ebd. S. 9, Hervorhebung des Autors.

19 Siehe z. B.: „En vérité, il n’y aurait pas d'exagération à dire que l'inventeur a découvert les moyens de fixer les images." François Arago: Protokoll der Sitzung vom 07. Januar 1839. In: Comptes rendus hebdomadaires des séances de l'Academie des sciences 8, 1839.

20 Diverse Äußerungen beschreiben die frühe Fotografie als eine spontane Selbsteinschreibung des Bildes, das auf der Mattscheibe der Camera obscura erscheint: „Cette découverte consiste dans la reproduction spontanée des images reçues dans la chambre noire." Joseph Nicéphore Niépce, Louis J. M. Daguerre: Bases du traité provisoire d'association Niépce - Daguerre, 14.12.1829, Russische Akademie der Wissenschaften, Sankt Petersburg, Ms 106 / K101, zit. nach http://www.archivesniepce.com/1-heliographie/1heliographie-par-les-lettres (Stand 5/2012); ,[...] and therefore, as the process presents us spontaneous$l y$ with a variety of shades of colour, it was thought best to admit whichever appeared pleasing to the eye, without aiming at an uniformity which is hardly attainable." Henry Fox Talbot: The Pencil of Nature, London 1844, S. 13 (Hervorhebungen des Autors). 
Effekt im Filmischen entspricht. In der Umgangssprache finden sich heute allerdings viele Beispiele, in denen das Einfrieren auch auf Fotografie angewendet wird. ${ }^{21}$

Mit einer Verkürzung der Belichtungszeiten und im Kontext der Chronofotografie bekommt der Begriff der Instantaneität zunehmende Bedeutung. ${ }^{22}$ Die Aufnahmen Eadweard Muybridges, Étienne-Jules Mareys, Ottomar Anschütz und Albert Londes sorgen dafür, dass deutlich wird, wie Fotografien (und in der Folge auch der Film) Raum und Zeit in distinkte sequenzielle Einzelbilder zerlegen, deren Schnitt eine gewisse Willkürlichkeit ausstrahlt und damit auf ein Einfrieren des Bildes hinweist. Die Bewegung wird willkürlich angehalten, was den Freeze-Effekt deutlicher macht als die idealisierte Bewegungsdarstellung eines besonderen Moments. ${ }^{23}$

Diese Art der Momentaufnahme markiert in der Fotografie einen eigenen Medieneffekt, auf den schon Walter Benjamin hingewiesen hat:

„Es ist ja eine andere Natur, welche zur Kamera als welche zum Auge spricht; anders vor allem so, dass an die Stelle eines vom Menschen mit Bewusstsein durchwirkten Raums ein unbewusst durchwirkter tritt. Ist es schon üblich, dass einer, beispielsweise, vom Gang der Leute, sei es auch nur im groben, sich Rechenschaft gibt, so weiß er bestimmt nichts mehr von ihrer Haltung im Sekundenbruchteil des, Ausschreitens. Die Photographie mit ihren Hilfsmitteln: Zeitlupen, Vergrößerungen erschließt sie ihm. Von diesem Optisch-Unbewussten erfährt er erst durch sie, wie von dem Triebhaft-Unbewussten durch die Psychoanalyse."

Der Freeze ist demnach ein medienreflexiver Moment, in dem sich das optisch Unbewusste zeigen kann.

Laura Mulvey betont die in der Stillstellung des Filmbildes liegende Verbindung zu den psychoanalytischen Theorien des Unbewussten:

„As stillness intrudes into movement, the image freezes into the ,stop of death', taking the aesthetics of cinema that leads back to pre-cinema, and to photographic and psychoanalytic theories. The blurred boundaries between the living and the not-living

21 Außerhalb des Metaphorischen werden analoge Filme tatsächlich gekühlt oder eingefroren, um sie länger haltbar zu machen.

22 Vgl. Phillip Prodger: Time Stands Still: Muybridge and the Instantaneous Photography Movement, New York 2003; André Gunthert: La conquete de l'instantané: Archéologie de l'imaginaire photographique en France (1841-1895). Ph.D. diss., École des Hautes Études en Sciences Sociales, Paris 1999. Zur Geschichte der apparativ verkürzten Belichtungszeit siehe auch Marta Braun: Picturing Time: The Work of Etienne-Jules Marey, Chicago 1992 und Jimena Canales: A Tenth of a second, Chicago 2009.

23 Wie es z. B. Henry Cartier Bresson mit seinen Bildern als „decisive moment“ beschreibt. 
touch on unconscious anxieties that then circulate as fascination as well as fear in the cultures of the uncanny. This shudder, however consciously experienced, is a symptom of the unconscious difficulty that the human mind has in grasping death and its compensatory capacity to imagine an afterlife. " 24

Hier wird auch ein zentraler Unterschied von Film und Fotografie adressiert, wie bei André Bazin, der den Film mit einem Mumifizierungsprozess verglichen hat: „Zum ersten Mal ist das Bild der Dinge auch das ihrer Dauer, eine sich bewegende Mumie.“25 Christian Metz hat diesen Gedanken eines untoten Zustands noch einmal erweitert:

\begin{abstract}
„Der Film gibt den Toten einen Anschein von Leben zurück, der zwar schwankend, schattenhaft und fragil ist, aber sogleich durch das Begehren des liebenden Publikums und sein drängendes Verlangen nach Stillung desselben verstärkt wird, während die Fotografie kraft der objektiven Suggestion ihres Signifikanten - Unbewegtheit und Stille - die Toten als Tote würdigt. ${ }^{26}$
\end{abstract}

\title{
IV. Die Zeit der Gewehrkugel
}

Der Freeze Frame öffnet einen Zwischenraum. Er hat im Gegensatz zum stillgestellten Bild der Fotografie eine bestimmte Dauer und ist damit endlich. Stillstellung ist allein in der Zeit erfahrbar. Bilder können also erst eingefroren werden, wenn sie vorher - als Bilder - bewegt waren. Die erstarrte Daphne in der Plastik Berninis wird nicht durch das Einfrieren eines Bildes erzeugt, sondern durch die Assoziation einer zu Stein gewordenen Figur. ${ }^{27}$

Im narrativen Kino wird der Freeze-Effekt oftmals am Ende eines Films eingesetzt, um die Handlung unaufgelöst in die simulierte Fotografie zu überführen, die im filmischen Kontext nichts von ihrer Zukunft weiß. ${ }^{28}$ Im Sinne dieses Einfrierens

24 Laura Mulvey: Death 24x a Second - Stillness and the Moving Image, London 2006, S. 23

25 André Bazin: Ontologie des fotografischen Bildes. In: Hartmut Bitomsky, Harun Farocki, Ekkehard Kämmerling (Hg.): Was ist Kino? Bausteine zur Theorie des Films, Köln 1975, S. 25.

26 Christian Metz: Foto, Fetisch. In: Hubertus von Amelunxen (Hg.): Theorie der Fotografie IV 1980-1995, München 2000, S. 345-355, hier S. 349f.

27 Wie sie schon von Ovid beschrieben wurde: „Ringsum hab’ er gesehn viel Bilder von Menschen und Tieren, die aus belebten in Stein gewandelt der Blick der Medusa“ (perque vias vidisse hominum simulacra ferarumque in silicem ex ipsis visa conversa Medusa), Metamorphosen, Vers 780.

28 Die unterschiedlichen Einsatzweisen des Freeze Frame können hier nicht detailliert besprochen werden. Siehe weiter dazu Sean Cubitt: The Cinema Effekt, Boston 2004, S. 212-215 und Diekmann, Gerling (s. Anm. 12). Bekannte Filme, die auf einem Freeze enden, sind: Les Quatre Cents Coups (François Truffaut, 1959), Butch Cassidy and the Sundance Kid (George Roy Hill, 1969), Thelma and Louise (Ridley Scott, 1991). 
von Bildern existieren zwei Arten der Stillstellung im Film: Einerseits die Standbildkopie, der schon erwähnte Freeze Frame, und seit einiger Zeit auch der Bullet Time genannte Effekt.

Im Freeze Frame wird das Bild angehalten bzw. durch Multiplikation des Einzelbildes in den zeitlichen Verlauf des Films übertragen. Im Time Slice $^{29}$ - ein älterer Begriff für die Bullet Time - wird der Moment der Aufnahme durch die Multiplikation der Kameras und damit der Perspektiven auf den Gegenstand sowie deren Übertragung auf den Film zu einer Bewegung im Raum im zeitlichen Fortschreiten des Films. ${ }^{30}$ In beiden Fällen handelt es sich um ein spatio-temporales Paradox, für das

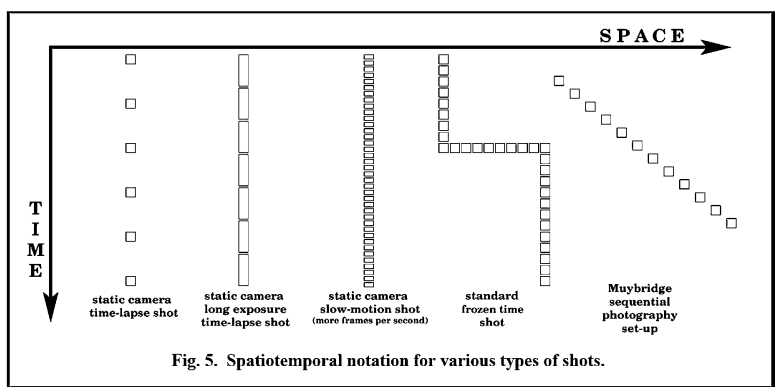

5: Mark J.P. Wolf: Raumzeitliche Kamera-Notation, 2006.

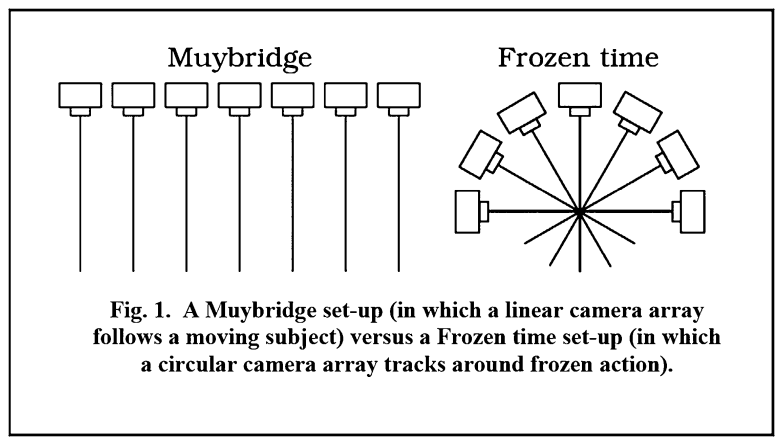

6: Mark J.P. Wolf: Notation der Kameraausrichtung, 2006. Mark J.P. Wolf in seinem Aufsatz zur Bullet Time eine grafische Notation findet. ${ }^{31}$ 入 Abb. 5+6

Anhalten der Aktion unter gleichzeitigem Bewegen des Materials ist ein wesentliches Prinzip des Einfrierens von Filmbildern, denn nur in dieser Differenz wird der gewünschte Effekt sichtbar. Die Bullet Time ist als paradoxale Struktur innerhalb des fortlaufenden Films imstande, die Zeit auszusetzen, den Raum aber weiter zu erschlieBen: Eine Figur wird im Raum arretiert und immobilisiert, zugleich aber innerhalb des Raums und der filmischen Zeit bewegt. $\boldsymbol{\lambda}$ Abb. 7-9 So können in der Bullet Time narrativ auch unterschiedliche Zeitökonomien parallel dargestellt werden: Eine Figur wird eingefroren, eine andere bewegt sich parallel in der Szene fort.

In vielen Computerspielen kann seit jüngerer Vergangenheit im sogenannten Fotomodus der Verlauf des Spiels in einer der Bullet Time ähnlichen Weise suspendiert werden, um sich vollkommen frei in der scheinbar eingefrorenen Szene zu

29 Siehe Anm. 12.

30 Vgl. Gerling (s. Anm. 12), S. 162.

31 Vgl.: Mark J. P. Wolf: Space, Time, Frame, Cinema - Exploring the possibilities of spatiotemporal effects. In: New Review of Film and Television Studies, 2006, 4 (3), S. 167-181, doi: 10.1080/17400300600981876. 


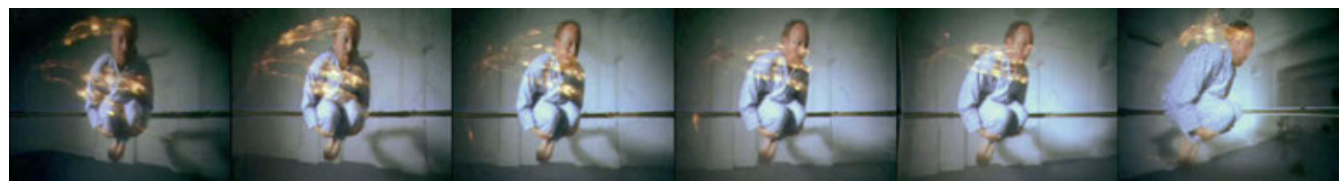

7: Montage des Autors aus Filmmaterial von Tim Macmillan.

bewegen. ${ }^{32}$ त Abb. 10 Auf diese Weise können In-Game-Fotos angefertigt werden, die unter Aussetzung des Spielverlaufs nicht den Bedingungen des Spiels ausgeliefert sind, sondern nur dem fotografischen Anliegen. ${ }^{33}$ Das Anhalten der Zeit ist auch hier Bedingung eines Studiums, einer intensivierten Wahrnehmung von Räumen und Gegenständen.

\section{Doppeltes Gefrieren}

Das sogenannte Einfrieren des Bildes hat grundsätzlich zwei Dauern: einerseits jene, die es innerhalb des Kontinuums Film einnimmt, andererseits diejenige, mit der das einzelne Bild belichtet wurde. Die unterschiedlichen Formen des Bewegtbildes haben aber jeweils eigene technische Grundlagen. Analoger Film, Video, digitaler Film/ Video und Computerspiel unterscheiden sich prinzipiell darin, Bewegung anzuhalten bzw. einzufrieren.

Im analogen Film wird ein Frame mehrfach kopiert und kann an das jeweils folgende oder vorausgegangene Bild räumlich und zeitlich anschließen. In der Wiederholung des analogen Frames wird aber trotzdem eine Bewegung des Films sichtbar bleiben, da sie materiell auf multiplen und unterschiedlichen Filmbildern basiert. Das typische Bildrauschen durch unterschiedliche Körnung, Staub und Verschleiß führt zwangsläufig zu einer Bewegung im Bild. Eine Bewegung, die digital in diversen Schnittprogrammen simuliert werden kann, um dem Film ein weniger hyper-reales Aussehen zu verleihen.

Ein Standbild im analogen Video lässt sich dagegen nur erzeugen, indem die Bewegung des Bandes gestoppt wird, der Abtastkopf des Recorders aber weiter rotiert. Anders als beim Film würde das Bild hier auch nicht durchbrennen, wenn es im heißen Projektor angehalten wird. Es entsteht aber auch im Video eine typische Bewegung im Bild, die aus dem Abtasten des rotierenden Abspielkopfes resultiert. Wie Raymond Bellour beschreibt, führte diese Möglichkeit des Videos zu neuen Praktiken bei der Lektüre eines Films: „Das Anhalten auf dem Bild bringt den Film in die Nähe des Buches, ist ein

32 Eine Technologie, die vom Grafikkartenhersteller Nvidia „Ansel“ (nach dem berühmten amerikanischen Landschaftsfotografen Ansel Adams) genannt wird: https:/www.nvidia.com/de-de/geforce/ geforce-experience/ansel/ (Stand 3/2021).

33 Vgl.: Sebastian Möring, Marco de Mutiis: Camera Ludica: Reflections on Photography in Video Games. In: Michael Fuchs, Jeff Thoss (Hg.): Intermedia Games - Games Inter Media: Video Games and Intermediality, New York 2019, S. 69-94. 
Durchblättern. Indem jedoch diese Geste gegen das ,natürliche“ Vorbeiziehen der Bilder ankämpft, bedeutet sie mehr als nur das: Ein Spiel, eine Umwandlung, ein Abschweifen..., eine abgeleitete Neuschaffung.“ ${ }^{34}$

Im digitalen Video/Film wird für die Erzeugung eines Standbildes ein Frame in einer digitalen Schnittsoftware identisch kopiert. Aber schon in der Aufnahme von digitalem Video sind Belichtungszeiten möglich, die im analogen Film nicht denkbar waren. Das Filmbild hat aufgrund seiner Wiederholrate von 24 Bildern pro Sekunde (Video: $25 \mathrm{BpS}$ ) in der Regel eine Belichtungszeit von 1/48 Sekunde. Da das Filmmaterial physisch bewegt werden muss, ist eine längere Belichtung nicht möglich und eine kürzere nicht üblich. So entsteht der typische Look eines Filmbilds: Das einzelne Bild ist in der Bewegung immer ein wenig unscharf.

Mit heutigen digitalen Kameras können bei einer Videoaufnahme, die im Prinzip nichts anderes als eine fotografische Serienaufnahme ist, sowohl sehr viel kürzere Belichtungszeiten als auch längere gewählt werden, theoretisch sogar längere, als das einzelne Filmbild im Abspielmodus dauert. Das erzeugt Effekte, die auch die Ästhetik des Freeze Frame verändern. Ist die Belichtungszeit z. B. sehr viel kürzer als $1 / 50$, z. B. 1/500, dann ist das Bild einer Bewegung im einzelnen Frame sehr

34 Raymond Bellour: Die Analyse in Flammen (Ist die Filmanalyse am Ende?). In: Montage AV, 1999, 8 (1), S. 19.

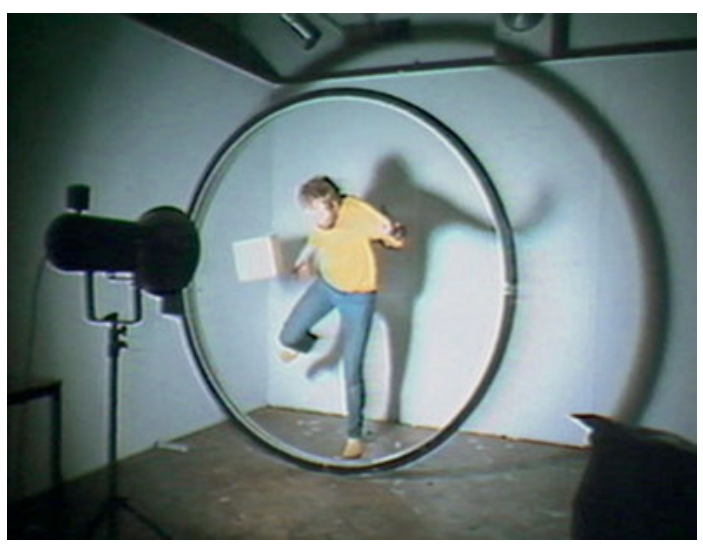

8: Tim Macmillan: Time-Slice Camera C 1982.

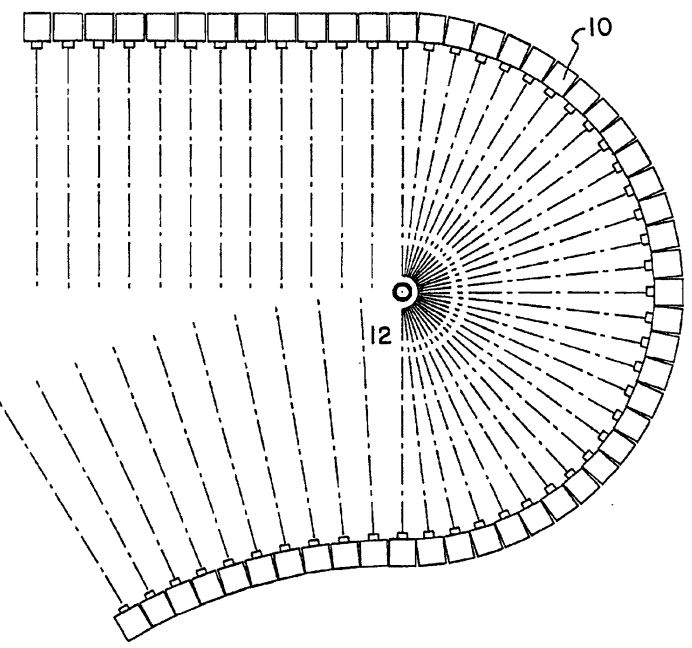

9: Schematische Skizze des Kameraaufbaus.

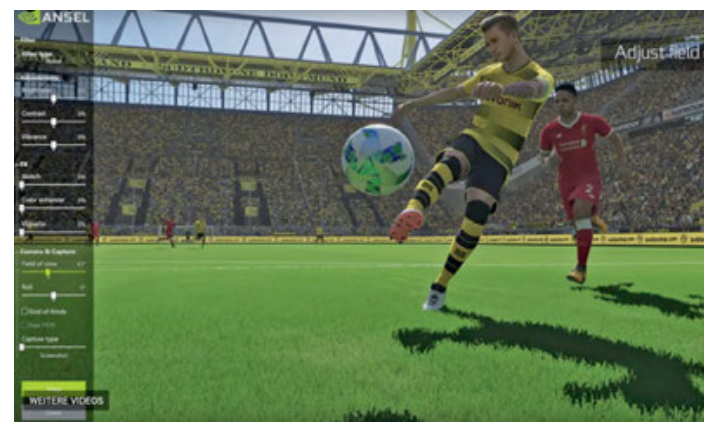

10: Nvidia: Ansel, Pro Evolution Soccer 2018, Videoeinstellungs-Interface. 


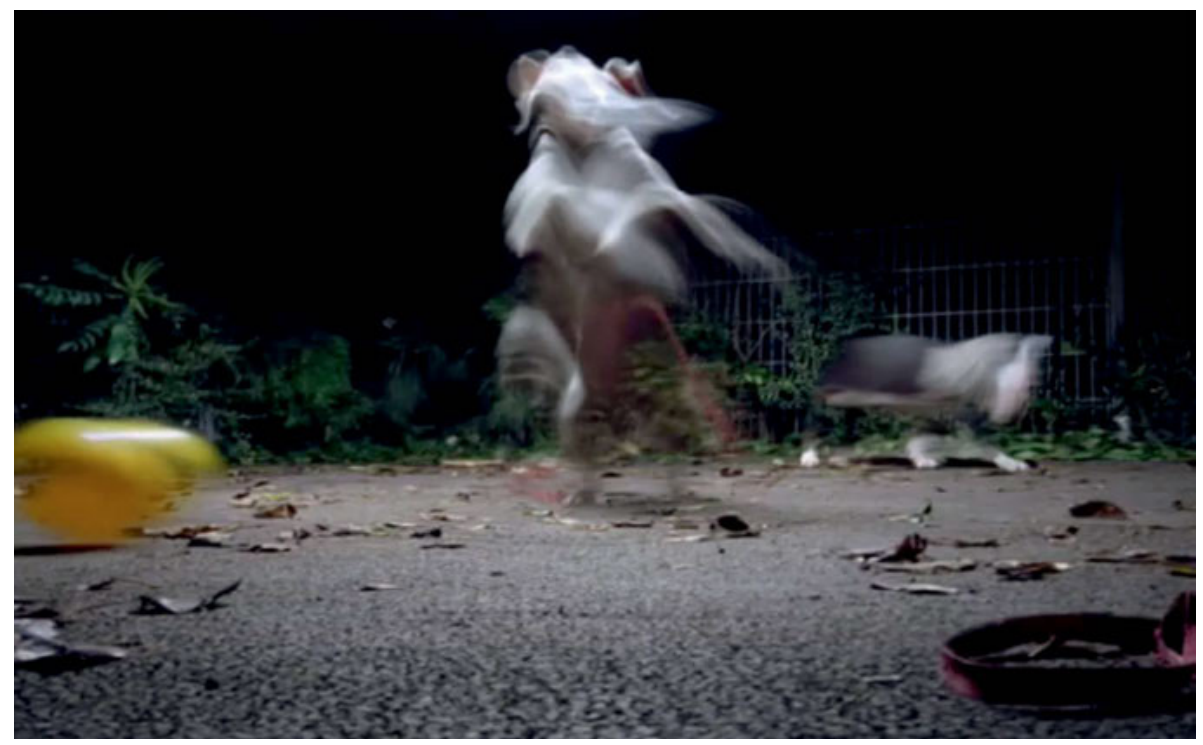

11: Chris Cunningham: Video-Still aus Werbevideo für die französische Telekommunikationsfirma Orange, 2007, 0:40 min., TC: 0:12.

viel schärfer als im ,traditionellen` Film und erzeugt so eine ungewohnte Schärfe bei bewegten Gegenständen oder auch bei Kamerabewegungen. ${ }^{35}$ Bei längeren Belichtungszeiten als 1/50 entsteht der gegenteilige Effekt. Die Bilder werden unscharf, beim Abspielen des Videos ist das Bewegtbild ungewohnt unscharf. Ein besonderer Einsatz dieser Unschärfe wird vom Videokünstler Chris Cunningham in einem Werbevideo für die französische Telekommunikationsfirma Orange vorgenommen. Er verbindet hier Aufnahmen im Modus der Bullet Time mit Langzeitbelichtungen. $\boldsymbol{\lambda}$ Abb. 11

Die Erscheinungsform des digitalen Videos verweist damit auf eine Grundbedingung des Films, nämlich, dass er - noch immer - aus unterschiedlichen aufeinander folgenden fotografischen Einzelbildern besteht und Stillstand nur als ausbleibende Bewegung simuliert werden kann. Bewegtheit ist Bedingung des Mediums. Das gilt für den digitalen Film bzw. das digitale Video in besonderer Weise: Nicht das (Film-) Material wird als moving image bewegt, sondern das Bild wird virtuell als re-moved image wiederholt.

35 Im Kontext des Kinos wurde diese Hyperrealität der digitalen Aufnahmetechnik, die Räume als Sets erkennen lässt und Figuren als deren Darsteller*innen, oftmals kritisiert. Vgl. Simon Rothöhler: High Definition - Digitale Filmästhetik, Berlin 2013, S. 41-58. 
Im Prozessieren ist kaum relevant, ob es sich um ein virtuell stehendes oder ein bewegtes Bild handelt. Das Bild kann heute mit sehr hoher Wiederholrate gezeigt werden, ${ }^{36}$ es bleibt aber immer noch ein geteiltes Bild, ein Schnitt in der Zeit, und steht damit im Digitalen ,materiell ' der Fotografie näher als dem Film. ${ }^{37}$ Das stehende Bild auf dem Display irgendeines digitalen Gerätes ist ein hochfrequent wiederholtes identisches Bild. Damit ist zumindest theoretisch ein Freeze möglich, der - anders als im analogen Film bzw. Video - vollkommen bewegungslos und störungsfrei stillsteht.

So wird auch der Screenshot eines Displays zu einem besonderen Freeze-Moment. In ihm wird die dauernde Bewegung auf dem Schirm und sogar die Operativität der bildlichen Zeichen aufgehoben, um das Zeugnis eines Screen-Moments als Datei abzulegen. ${ }^{38}$ Ein Einfrieren, das insbesondere auf Smartphones zu seltsamen Verwechslungen führen kann. Wer einen Screenshot bedient, der identisch ist mit dem operativen Interface, kann verwundert feststellen, dass als eingefrorenes Bild dieses Interface nicht $\mathrm{zu}$ bedienen ist.

Auch bei abstürzenden ${ }^{39}$ Computern wird gelegentlich davon gesprochen, dass der Bildschirm einfriert, obwohl der Grund dafür wahrscheinlich eher ein heißgelaufener Prozessor ist. Hier ist das Einfrieren eine willkürliche Unterbrechung des always on ${ }^{40}$ das auf eine besondere Form der Abhängigkeit des Menschen von Computern und den mit ihm verbundenen Infrastrukturen hindeutet. Mit diesem Phänomen schließt sich der Kreis wieder zum Beginn des Textes, denn das Einfrieren einer Kachel im Panel der Videokonferenz zeigt das temporäre Aussteigen aus einer privilegierten, abgeschirmten Sicherheit, die Unterbrechung einer medial vermittelten Co-Präsenz. Friert im Raster der Videokonferenz das eigene Bildnis ein, ist die beteiligte Person auch in sozialer Hinsicht für den entsprechenden Moment kaltgestellt.

36 Bis zu $1.000 \mathrm{~Hz}$, wobei die materielle Basis - die Bildaufnahmerate - eines Videos oder Films im Kunstund Unterhaltungsbereich immer noch zwischen 24 und 72 Bilder pro Sekunde liegt; dementsprechend oft wird dann dasselbe Bild wiederholt. In wissenschaftlichen oder auch militärischen Einsatzgebieten sind sehr viel höhere Bildaufnahmeraten möglich (bis zu 90.000 Hz), wie z. B. bei Hochgeschwindigkeits-Infrarotkameras.

37 Mit Henri Bergson argumentiert, nähern wir uns zwar einer ungeteilten Bewegung an - der Wirklichkeit -, werden sie aber auf diesem Wege nie erreichen: „In Wirklichkeit gibt es niemals eine wirkliche Unbeweglichkeit, wenn wir darunter eine Abwesenheit von Bewegung verstehen. Die Bewegung ist vielmehr die Wirklichkeit selbst.“ Henri Bergson: Denken und schöpferisches Werden. In Friedrich Kottje (Hg.): Aufsätze und Vorträge, Hamburg 1993, S. 162ff.

38 Vgl:: Winfried Gerling: Photography in the digital - Screenshot and in-game photography. In: photographies, 2018, 11 (2-3), S. 149-167, hier S. 160.

39 Engl.: crash.

40 Vgl. Jan Distelmeyer: Kritik der Digitalität, Wiesbaden 2021, S. 114-122. 